
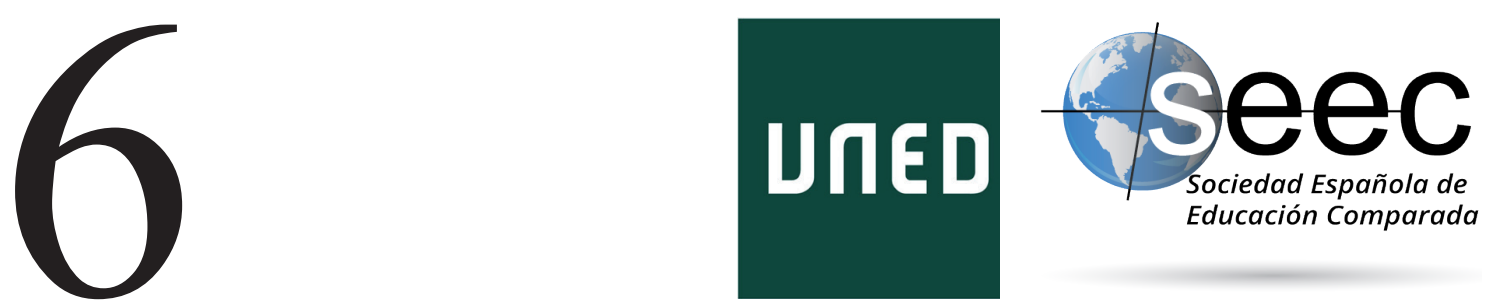

\title{
Estrategias educativas para generar seguridad a los desplazados en España
}

Educational strategies to generate security for displaced people in Spain

\section{Ma del Pilar Quicios García*; Ana Ma Huesca González $^{* *}$; Patricia González Ocejo ${ }^{* * *}$}

DOI: $10.5944 /$ reec.35.2020.25216

Recibido: 4 de julio de 2019 Aceptado: 19 de noviembre de 2019

\footnotetext{
*Ma del Pilar Quicios García: Profesora Titular de Universidad en el Departamento de Teoría de la Educación y Pedagogía Social de la Facultad de Educación de la UNED y Directora de la Red de investigación internacional Prevención educativa y sociocomunitaria del riesgo social (PEYSDRA). Datos de contacto: E-mail: pquicios@edu.uned.es

**Ana Ma Huesca González: Profesora del Departamento de Sociología y Trabajo Social en la Facultad de Ciencias Humanas y Sociales de la Universidad Comillas de Madrid. Datos de contacto: E-mail: ahuesca@comillas.edu ***Patricia GonZÁlez OcEJo: Maestra funcionaria de carrera con las especialidades de francés, inglés, pedagogía terapéutica y audición y lenguaje en colegios dependientes de la Consejería de Educación de la Junta de Castilla y León (España) y miembro investigador de la red de investigación internacional PEYSDRA. Datos de contacto: E-mail: patricia.gonoce@educa.jcyl.es
} 


\title{
Resumen
}

Las poblaciones desplazadas están formadas por emigrantes, inmigrantes, asilados, refugiados y apátridas. Cada una de estas personas abandona su país y sus coordinadas vitales por motivos diferentes. El objetivo que comparten es la búsqueda de seguridad. Seguridad vital, jurídica o económica. El objetivo perseguido no siempre se alcanza. Este choque entre expectativa y realidad sume en una permanente incertidumbre a gran número de los desplazados. Los más vulnerables pueden desarrollar un síndrome específico del inmigrante con estrés crónico y múltiple (el Síndrome de Ulises). Este desajuste psicosocial, desde 1985, se aborda transdisciplinariamente desde la medicina, la psicología, la educación y el trabajo social. En este artículo discursivo-divulgativo se transfieren algunos resultados de dos investigaciones que combinan la metodología cuantitativa y la cualitativa. El objetivo 4 de la Investigación CSO2016-77549-P (Plan Nacional de I+D, AEI/FEDER, UE) y la prospectiva de la investigación Alfabetización para la integración social de inmigrantes y refugiados adultos en España (Programa Ayuda Europa-Investigación. UNED-Banco Santander, 2017/2019). Los resultados de estas investigaciones del campo de la educación, la sociología y la educación social indican algunas de las estrategias educativas y sociocomunitarias que pueden ayudar a los desplazados llegados a España a rebajar su nivel de incertidumbre y a acrecentar su sentimiento de seguridad. Son estrategias que puestas en práctica evitarán medicalizar a personas que no necesitan fármacos. Personas que sufren un desajuste psicosocial que necesita apoyo emocional, terapia psicoeducativa, asesoramiento jurídico y trato empático por la sociedad del país de acogida. La ejecución de estas estrategias ayudará a fortalecer emocionalmente a cada uno de los desplazados pudiendo reconducir la expresión conductual, cognitiva y psicofisiológica del sistema de respuesta dado a las emociones negativas percibidas.

Palabras clave: refugiado; derechos humanos; derecho a la educación; dificultad de aprendizaje; integración escolar

\begin{abstract}
Displaced populations are made up of emigrants, immigrants, asylees, refugees and stateless persons. Each of these people leaves their country and their vital attachments for different reasons. The aim they share is the search for security, either vital, legal or economic security. The objective pursued is not always achieved. This clash between expectation and reality adds up to a permanent uncertainty to a large number of the displaced people. The most vulnerable can develop a specific immigrant syndrome with chronic and multiple stress (Ulysses Syndrome). This psychosocial mismatch, since 1985, is tackled interdisciplinarily from medicine, psychology, education and social work. In this discursive-informative article some results of two investigations that combine quantitative and qualitative methodology are transferred. Objective 4 of Research CSO2016-77549-P (National R\&D Plan, AEI / FEDER, EU) and the prospective research Literacy for social integration of immigrants and adult refugees in Spain (EuropeResearch Assistance Program. UNED-Banco Santander, 2017/2019). The results of these investigations in the field of education, sociology and social education indicate some of the educational and socio-community strategies that can help displaced people arriving in Spain to lower their level of uncertainty and increase their sense of security. They are strategies that put into practice will avoid medicalizing people who do not need drugs. People who suffer from a psychosocial imbalance that needs emotional support, psychoeducational therapy, legal advice and empathic treatment by the society of the host country. The execution of these strategies will help to emotionally strengthen each one of the displaced people, being able to redirect the behavioral, cognitive and psychophysiological expression of the response system given to the perceived negative emotions.
\end{abstract}

Keywords: Prevention through education; displaced people; psychosocial maladjustment; emotional intelligence; Ulysses syndrome; security 


\section{Introducción}

Las poblaciones desplazadas, dependiendo del motivo que origine su viaje reciben diferentes nombres. Nombres que van desde inmigrantes a emigrantes, asilados, refugiados o apátridas. Con independencia del motivo que haya originado el desplazamiento de cada uno de estos colectivos, todas estas personas buscan seguridad. Entre otras, seguridad vital al tratar de preservar la vida (Lara, 2019). Seguridad jurídica al ansiar ejercer los derechos humanos que ostentan todas las personas (Huesca, 2009, 2016). Seguridad económica para ellos y sus familias al anhelar una mejora en su calidad de vida (Vauclaire y Bratanova, 2016). Para disfrutar cualquiera de esas seguridades llegan a un país diferente al suyo ${ }^{1}$. Unos lo hacen cumpliendo la legislación migratoria internacional o la que rige en el país de destino. Son los desplazados administrativamente regularizados. Otros, acceden a esos mismos países sin cumplir tales requisitos convirtiéndose legalmente en desplazados no regularizados o, peor todavía, en un simpapeles o en un ilegal.

Los desplazados regularizados pueden recibir atención de la sociedad del país de acogida. Esa ayuda, por muy pequeña que sea, les permite sobrellevar someramente los problemas que conlleva el acceso a una nueva sociedad, cultura y vida. Caso muy distinto es el de las poblaciones no regularizadas. Éstas se encuentran con pocas opciones de trabajo y con riesgo de ser expulsados del país de llegada. Ante esta situación, una proporción nada desdeñable de ellos enferma. Desarrolla un malestar emocional (Vallejo-Sánchez y Pérez-García, 2016), una enfermedad emocional ${ }^{2}$ (García Carrasco, 2006) o, un desajuste psicosocial exclusivo de la población desplazada. Ese desajuste psicosocial está motivado por la permanente incertidumbre a la que se ven expuestos (Navarro, 2015).

Síndrome de Ulises es el nombre que recibe esta patología descubierta o, al menos, sistematizada en 1985 por el psiquiatra Joseba Achotegui (la Comunidad científica no se aventura a afirmar rotundamente que sea él el descubridor de este síndrome psicosocial). Motivos no le faltan. Quizá el más fundamentado sea que con anterioridad a la indudable, reconocida e importante aportación psicológica, psiquiátrica y sociocomunitaria de Achotegui [(2000), (2002a), (2002b), (2003a), (2004a), (2004b), (2009), (2017); Achotegui, Lahoz, Marxen, Espeso, (2005); Achotegui, López, Morales, Espeso, Achotegui, (2015)] la bibliografía médica ya recogía en sus manuales de psiquiatría referencias a similares sintomatologías - aunque ciertamente que de una forma parceladacon los nombres de estrés por choque cultural (Oberg, 1960) y estrés por aculturación (Berry, 1980, 1990, 1997).

El Síndrome de Ulises -síndrome del inmigrante con estrés crónico y múltiple-se define como un desajuste mental emergente que contiene síntomas comunes tanto al estrés por choque cultural como al estrés por aculturación (Achotegui y Jayme, 2016). Este nuevo síndrome se desencadena, principalmente, entre dos tipos de desplazados: aquellos no regularizados y, en menor medida, entre los desplazados legalizados con permiso de residencia y trabajo que han perdido su legalización o están a punto de perderla (Delgado, 2013).

$1 \quad$ Resultados parciales del objetivo 4 de la Investigación del Plan Nacional de I+D ref. CSO201677549-P (AEI/FEDER, UE) que pretende mostrar cómo las incertidumbres vitales asociadas a la vida cotidiana afectan al sentimiento de inseguridad en España.

2 El concepto de enfermedad emocional indica que la dinámica de la sensibilidad puede alterarse como consecuencia de influencias ambientales. 
La intensidad del Síndrome también varía entre los distintos tipos de desplazados. Los desplazados no legalizados lo sufren con mayor amplitud e intensidad, con más desconsuelo y mayor desesperanza. El desajuste psicosocial de ambos tipos de desplazados clava sus raíces en sus esperanzas rotas. Cristina Cortés (2011), coordinadora de la Jornada sobre Inmigración y Salud del Hospital General de L'Hospitalet de Llobregat (Barcelona) mantiene:

«el desarraigo, el estar alejados de la familia y no cumplir las expectativas que tenían cuando llegaron a España son las principales causas de estos problemas de salud mental que se han incrementado con la crisis económica» (citado en Rodríguez, O., 1 de marzo de 2011, www.tomai.es).

Las víctimas del Síndrome de Ulises salen de sus países estresados, ilusionados, ansiosos o esperanzados. Se despiden de sus familias. Cierran sus hogares. Asumen fuertes deudas para pagar los viajes (en algunos casos pagan mordidas a mafias que les introducen ilegalmente en el país de destino). La determinación de abandonar sus referencias vitales, en sí misma, es un detonante de enfermedad emocional, malestar emocional o desajuste psicosocial que aún no se ha manifestado aunque esté latente (Lara, et al. 2017).

El Síndrome de Ulises tiende a agravarse en intensidad de manera paralela a la distancia existente entre el sueño acariciado y la realidad encontrada. Los desplazados afectados por el Síndrome de Ulises viven entre cuatro dramas iniciales: el primer y segundo drama, se manifiesta más entre los desplazados económicos. Estos dos dramas se producen porque estos trabajadores no pueden ejercer sus actividades laborales en España y tienen que seguir enviando dinero a sus países. «iYo no quiero comer de gratis! iQuiero trabajar! iSoy matrona! iNecesito trabajar de cualquier cosa! iMis hijos tienen que comer diariamente y yo tengo que pagar la deuda contraída para llegarme a España!» son frases que se repiten en los grupos de discusión celebrados en la investigación de corte cualitativo sobre la que se asienta parte de este artículo de transferencia de investigación ${ }^{3}$.

El tercer y cuarto drama pasa por la falta de comunicación veraz. Ni los desplazados económicos ni los refugiados, ni siquiera los apátridas, pueden indicar a sus familias su fracaso. Su drama se vive en silencio o se enmascara en una realidad inventada.

Desde 1985 Achotegui, se involucró en el estudio de este desajuste psicosocial. El Síndrome de Ulises comenzó a estudiarse transdisciplina y complementariamente desde la medicina, la psicología, la educación y el trabajo social entre otras disciplinas. Algunos de los médicos interesados en su estudio fueron González de la Rivera (2005), Vázquez (2005), Cortés (2008), Bennegadi (2008), Espeso (2009). También son conocidas las aportaciones psicológicas de Castro (2010), los estudios pedagógicos de Quicios (2008, 2010) y las actuaciones desde el trabajo social de Moreno (2006), Huesca, Giménez y Quicios (2018).

La administración española también lo abordó desde sus fuentes de I+D financiando varios proyectos de investigación sobre esta temática. Algunos de ellos fueron I+D SEJ 2005-09170-CO4-01 o Prevención y detección precoz del Síndrome de Ulises (2006). La sanidad y la Academia organizaron congresos internacionales sobre la temática. La psiquiatría, como ciencia directamente implicada en el problema, ha profundizado en la temática publicando artículos profesionales. La ciudadanía, en general, se ha involucrado

3 Alfabetización para la integración social de inmigrante y refugiados adultos en España (Programa Ayuda Europa-Investigación. UNED-Banco Santander, 2017/2019). 
en este triste devenir saltando, incluso, una temática tan específica de la sanidad, la psiquiatría, la psicología y la educación a la prensa diaria (Gallego-Díaz, 20/o9/2011, El País. Sociedad).

Las conclusiones a las que han llegado - transdisciplinariamente- todos los estudiosos del tema, han confluido en un punto común: prevenir el síndrome de Ulises incumbe tanto a médicos como a educadores:

«el Síndrome de Ulises se halla inmerso en el área de la prevención sanitaria y psicosocial más que en el área del tratamiento. .../... La intervención tendrá por objeto evitar que las personas que padecen este cuadro acaben empeorando y lleguen a padecer un trastorno mental estándar (por lo que el trabajo sobre el Síndrome atañe no tan solo a psicólogos o psiquiatras, sino a trabajadores sociales, enfermería, educadores sociales...etc.)» (Achotegui, 2008: 24).

Espeso Montagud que investiga, desde el ámbito de la medicina, el Síndrome de Ulises en niños y jóvenes, en el 15 Congreso Mundial de Psiquiatría celebrado en Buenos Aires en septiembre de 2011 concluyó: «estas poblaciones precisan una acción psicosocial que le ayude a hacer frente a la situación de dificultad extrema en la que se encuentra». Reiterando: «es importante no patologizar ni medicalizar estos casos, sino buscar la forma de proporcionales apoyo emocional, psico-educativo y asesoramiento jurídico». (Citado en Gallego-Díaz, 20/o9/2011, El País. Sociedad).

En el Congreso Internacional de Inteligencia Emocional celebrado en Las Palmas de Gran Canaria en diciembre de 2008 se indicaba que la educación, con una visión preventiva, quiere anticiparse a la aparición de este Síndrome y que su labor se está centrando en informar, instruir y fortalecer emocionalmente a estos colectivos reconduciendo la expresión conductual, cognitiva y psicofisiológica del sistema de respuesta dado a las emociones que perciben (Vallés y Vallés, 2003).

\section{Acercamiento al Síndrome de Ulises}

En medicina, un síndrome es un cuadro clínico o un grupo significativo de síntomas y datos semiológicos que concurren a la vez con variada etiología. En el presente artículo se entiende por Síndrome de Ulises, desde el punto de vista socioeducativo, el conjunto de síntomas que padecen los desplazados no regularizados, exhaustos de luchar por sobrevivir en el país de acogida sin obtener resultados en esa lucha.

Los síntomas que suelen mostrar pertenecen al ámbito de la salud mental pero se manifiestan a través de dolencias físicas, psíquicas y psicosomáticas. Achotegui (2003) clasificó la sintomatología presentada por los pacientes por él atendidos en cuatro áreas. Éstas son:

1) Síntomas del área depresiva manifestadas a través de:

- Tristeza. Esta tristeza es la respuesta personal a dos aspectos frustrantes y dolorosos para el desplazado. Por un lado responde al fracaso de la aventura emprendida y por otro a la situación de indefensión que como trabajador foráneo le embarga.

- Culpa de doble naturaleza. Culpa paranoide con grave temor a un castigo por haber abandonado sus coordinadas vitales y culpa depresiva. 
- Ideas de muerte entre los adolescentes. Los desplazados adultos que han tomado la decisión de desplazarse (desplazados de primera generación) no desean morir. Al contrario, tienen deseos de vivir para ver cumplido el objetivo de su desplazamiento.

- Llanto o gemidos, dependiendo de la cultura a la que pertenezca el desplazado.

2) Síntomas del área de ansiedad manifestadas a través de:

- Tensión y nerviosismo debido al enorme esfuerzo vital y psicosocial mantenido en el tiempo.

- Preocupaciones excesivas y recurrentes. Estos pacientes acumulan múltiples sentimientos contrapuestos.

- Irritabilidad, generalmente, solo entre los menores.

- Insomnio causado por la tensión emocional y las múltiples preocupaciones a las que deben encontrar respuesta.

3) Síntomas del área de somatización manifestadas a través de:

- Cefaleas de tipo tensional en la zona frontal de la cabeza y en las sienes.

- Fatiga y falta de energía.

- Somatizaciones osteomusculares con contracturas musculares de espalda y articulaciones. Estas contracturas están originadas por el inmenso estrés sufrido.

- Algunas molestias abdominales o torácicas

4) Síntomas del área confusional manifestadas a través de:

- Fallos de memoria y de atención.

- Desorientación temporal.

- Huida de la realidad al no saber distinguir las mentiras inventadas para ocultar el drama vivido diariamente. La realidad vivencial es negada y redibujada creativamente.

La intensidad de esta sintomatología se clasifica en parcial o completa dependiendo del número de estresores que soporten los pacientes (Achotegui, 2003, 2004). Generalmente los afectados por este desajuste psicosocial se enfrentan a cuatro estresores. El primero proviene de la soledad obligada en la que tienen que vivir. Los desplazados carecen de red social de apoyo. Se encuentran solos y esa profunda soledad les asusta (Navarro-Lashayas, 2018). No confían en nadie y esa desconfianza les perjudica. No saben cómo comunicarse ni con quien hacerlo y esa desinformación les daña. Se ven obligados a vivir entre muchas personas pero dentro de esa aparente compañía, tienden a vivir solos. Sin nadie. Sobreviven rompiendo la lógica aristotélica del ser humano como ser eminentemente social. 
El segundo estresor surge al convivir en un continuo sentimiento de fracaso. La derrota nace al no poder conseguir un trabajo. En el caso de que el desplazado económico haya conseguido un mísero trabajo, también se origina cierto descalabro emocional al no lograr mejorar el precario trabajo que inicialmente se ha conseguido. Si a ese segundo estresor se le une alguna otra dificultad mantenida en el tiempo, el desplazado, comienza a caer en las redes del temido Síndrome (Quercetti, 2017).

El tercer estresor guarda una doble relación con el mundo laboral y con la feroz lucha por sobrevivir en unas condiciones vitales auténticamente infrahumanas. Muchos desplazados aquejados por el Síndrome de Ulises confiesan verse obligados a ejercer trabajos de alto riesgo por un mísero, oculto y discontinuo salario no superior a ocho o diez euros al día. La precariedad laboral se une al hacinamiento vital en infraviviendas de las que usan - cada uno de ellos- un máximo de dos metros cuadrados. Estos cubiles se denominan pisos patera. Están ubicados en barrios conflictivos y su estado de conservación es inexistente. Los desplazados más desfavorecidos no pueden ocupar un lugar en un piso patera, han de conformarse con dormir en las conocidas camas calientes por un periodo no superior a ocho horas diarias o pernoctar compartiendo espacios en sofás o en jergones con desconocidos compañeros de sábanas.

El cuarto estresor nace del miedo físico, emoción primaria negativa que implica inseguridad para soportar una posible amenaza próxima y que consigue paralizar física, psíquica, mental y afectivamente al desplazado aquejado de desajuste psicosocial. La suma de estos cuatro estresores forja el panorama socioafectivo que tiene que afrontar el desplazado (administrativamente ilegal) más vulnerable y desprotegido (Reig-Botella, Clemente, Sangiao, 2018).

\section{La educación ante el Síndrome de Ulises}

El Síndrome de Ulises, siendo exhaustivos, no puede ser considerado una depresión, ni una psicosis, ni un trastorno adaptativo. Es decir, no puede afirmarse que se trate de ninguna patología ni de ninguna enfermedad. El Síndrome de Ulises es un conjunto de síntomas provocado por un cúmulo de circunstancias sociales o ambientales que colocan al desplazado, no regularizado, en una difícil situación vital en el fino límite que separa la salud de la enfermedad. Esa es la opinión de muchas autoridades sanitarias.

Achotegui en el 15 Congreso Mundial de Psiquiatría (Buenos Aires, septiembre del 2011) declaraba: «tratarles como enfermos [a los afectados por el Síndrome de Ulises] es inadecuado, provoca un gasto innecesario para el sistema y no les ayuda a mejorar su estado» (citado en Gallego-Díaz, S. 20/09/2011, El País. Sociedad). Rachid Bennegadi, psiquiatra y antropólogo (director de investigación del Centro Françoise Minkowska de Paris) con más de 60 años de experiencia y más de 10.000 consultas anuales afirma:

«este síndrome no es una patología sino las consecuencias de las condiciones de presión extrema en las que las personas hacen su migración» (Citado en Espar 14/06/2008. Síndrome de Ulises, la enfermedad de los invisibles. El País, En Portada. P. 2).

Al descartar que el Síndrome de Ulises pueda considerarse una enfermedad exclusivamente fisiológica, cabe la opción de calificarle de problema sociológico abordable desde la Educación (concretamente desde la Pedagogía Social, desde la Pedagogía de la socialización y desde la Educación Social). La Educación, a través de la intervención 
sociocomunitaria, puede intentar prevenir el Síndrome. Incluso, puede rebajarlo - si ya se ha desencadenado-. Para llevar a efecto esta tarea, la Educación, entendida como un proceso perfectivo en sentido amplio, es decir, un proceso optimizador no circunscrito solamente al ser individual, sino prolongable a la sociedad en general, tiene que exigir al poder legislativo la regularización de la situación de estos colectivos. La Educación Social, así, propondrá dos conjuntos de acciones para prevenir o paliar la intensidad del Síndrome de Ulises, a saber:

1) Medidas dirigidas a desplazados regularizados que han cotizado a la Seguridad Social, han perdido su puesto de trabajo y han consumido la prestación por desempleo así como la prórroga de esta prestación. Siempre que el desplazado esté de acuerdo con la medida se podrá:

- Facilitar al trabajador inactivo un billete gratuito para retornar a su país de origen.

- Certificar, oficialmente, el desempeño del puesto de trabajo que haya realizado legalmente en España, su categoría profesional y su periodo de actividad.

- Crear, por nichos laborales, un censo de población desplazada formada, cualificada, capacitada. Esos censos laborales servirían de demanda de empleo permanente para estos trabajadores aunque residiesen en otros países.

- Redactar al trabajador inactivo una carta de trabajo preferente para ejecutar el retorno a nuestro país y se requieran trabajadores de su perfil laboral y su categoría profesional.

2) Medidas dirigidas a desplazados no regularizados que hayan perdido su puesto de trabajo en el mercado laboral opaco. Contando siempre con el beneplácito del trabajador se podrá:

- Facilitarle, de manera gratuita, un billete de retorno a su país de origen.

- Certificar, oficialmente, su estancia de forma no regularizada en España.

- Crear un censo de población inmigrante no cualificada que sirva de demanda de empleo permanente para estos trabajadores.

- Redactar al trabajador inactivo una carta de trabajo preferente para ejecutar el retorno a nuestro país y pueda demostrar, fehacientemente a las autoridades españolas el haber adquirido formación suficiente en su país de origen.

- Firma, por parte del desplazado no legalizado, de un documento en el que se comprometa a no retornar de manera ilegal a este país bajo pena de ser repatriado forzosamente a su país de origen y asumir el coste de todos los pasajes aéreos abonados por el Gobierno de España o las Comunidades autónomas para sus sucesivos retornos. 
Con estas actuaciones se está haciendo frente, globalmente, a los estresores dos, tres y cuatro. Los trabajadores desplazados que se acogieran a ellas podrían volver a sus países de origen sin sentimiento de fracaso, serían acogidos en sus comunidades sin ser estigmatizados y tendrían la posibilidad de volver cuando la situación socioeconómica lo permitiera. El coste económico para este país sería sumamente bajo, el ahorro que conllevaría en gasto social sería importante, el beneficio social entre la población desplazada y autóctona nada desdeñable y la imagen internacional del país muy saludable.

A su vez esta medida serviría para realizar una labor de educación social entre la población española que se ha servido, de forma directa o indirecta, de esa mano de obra cuando el país la requería. Educaría colectivamente en la asunción de responsabilidades y en el respeto a la dignidad personal del conciudadano. Esa acción de educación colectiva consistiría en revertir una serie de beneficios necesarios hacia las personas que han dejado en la sociedad su fuerza de trabajo. Educaría en deontología social al desterrar la idea existente, en estos momentos, de desechar a las personas que ya no resultan productivas o rentables. El legislar la manera de repatriarles, voluntariamente a sus países en condiciones nada desfavorables sería una manifestación de ética social, de equidad comunitaria hacia el trabajador foráneo y un posible germen de una justicia equitativa colectiva.

El Gobierno de España, a través del RDL 4/2008 de 19 de septiembre y del RD 1800/2008 de 3 de noviembre intentó, sin gran éxito, algo similar. Legisló un Plan de retorno voluntario consistente en el abono acumulado y de forma anticipada de la prestación contributiva por desempleo a trabajadores extranjeros no comunitarios que retornaran voluntariamente a sus países de origen. Su filosofía de base fue acertada pero las condiciones de acceso al Plan sumamente restrictivas tanto en lo referente a la nacionalidad de los beneficiarios ${ }^{4}$ como a las condiciones sociolaborales que debían cumplir para acogerse a él 5 .

\section{Educación social del inmigrante desolado}

Esbozada una actuación legislativa de raigambre socioeducativa para la situación en la que viven estos desplazados urge formarles en el manejo y control de las emociones que le produce su situación personal. Para ello la Educación ha ideado una serie de estrategias de autocontrol emocional.

«El control de las emociones.../... supone poseer una serie de habilidades que permiten a la persona hacerse cargo de la situación, tomar decisiones entre alternativas posibles y reaccionar de manera controlada ante los diversos acontecimientos de la vida» (Vivas, Gallego y González, 2006: 31).

4 Sólo podrían acogerse a él aquellos inmigrantes sin nacionalidad española provenientes de Andorra, Argentina, Australia, Brasil, Canadá, Chile, Colombia, Ecuador, E.E.U.U., Federación Rusa, Filipinas, Marruecos, Méjico, Paraguay, Perú, República Dominicana, Túnez, Ucrania, Uruguay y Venezuela.

$5 \quad$ Ser ciudadano de un país con convenio bilateral con España en materia de Seguridad Social, tener residencia legal en España, estar inscrito como demandante de empleo en el INEM o en los Servicios Públicos de Empleo de las distintas Comunidades Autónomas, encontrarse en situación legal de desempleo como consecuencia de la extinción de la relación laboral, tener reconocida una prestación por desempleo de nivel contributivo sin compatibilizarla con trabajo a tiempo parcial, retornar exclusivamente al país del que tenga la nacionalidad y no tener prohibida la salida de España por la Ley de Extranjería. 
Este tipo de inmigrante tiene que aprender a aislar los efectos de cada uno de los cuatro estresores a los que se enfrenta. Su lema vital habrá de ser «divide y vencerás» afrontando estresor a estresor de manera independiente. Reducir la intensidad de un solo estresor lleva acompañado un segundo beneficio que es la disminución del estrés acumulado que acompaña la vida diaria del desplazado (Salaberria y del Valle, 2017). Esa lucha por huir de la cronicidad del estrés acumulativo permitirá el nacimiento de un tercer aspecto beneficioso como es la incipiente sensación de control de su propia existencia superando el tercer y cuarto estresor.

Luchando por superar el estresor del fracaso, rebajando el nivel de estrés acumulado y comenzando a controlar su vida, el aquejado por el Síndrome de Ulises se sentirá capaz de relacionarse con los demás, crearse una mínima red social de apoyo, (rebajando el estresor uno), enfrentarse a los estresores clásicos de diferencia idiomática, paisajística, cultural y social que, generalmente, son plenamente asumibles por todo tipo de desplazados.

Con esa seguridad personal aumentará su calidad de vida al disminuir la somatización de sus dolencias y así acudirá al Centro de Salud para ser atendido en sus auténticos padecimientos fisiológicos, si es que sigue padeciéndolos. En los Centros de Salud ya se trabaja con estas poblaciones de manera interdisciplinar, es decir, sin perder de vista la finalidad educativa de las consultas médicas. Julia Moreno, trabajadora social del Equipo de salud mental de Médicos del Mundo de Aragón e investigadora del proyecto Prevención y detección precoz del Síndrome de Ulises (2006) afirma:

«[el proyecto]Procura tratamiento psicosocial para estos emigrantes basado en la orientación e información sobre los recursos de apoyo social (contacto con asociaciones, grupos, cursos, etc.) y asesoramiento legal. Paralelamente se interviene sobre los síntomas con una leve psicoterapia que ayude a la contención emocional y a clarificar la confusión. Y cuando existen síntomas paralizantes, como el insomnio o las crisis de ansiedad, se suelen administrar fármacos que alivien de forma temporal.» (Citado en Espar, 14/06/2008. Síndrome de Ulises, la enfermedad de los invisibles. El País, En Portada. P.2).

No todos los profesionales sanitarios de la red primaria están capacitados para entender la situación socioafectiva de estas poblaciones y, en algunos casos, en su inoperancia pueden desmoralizar más que ayudar a los pacientes a que clarifiquen la génesis de los problemas físicos que les han llevado a las consultas médicas (Chávez y Alava, 2019). Para no entorpecer - la labor educativa desplegada con estos pacientes desplazados por otros profesionales- la Educación también realiza una labor de orientación con el personal sanitario puesto que «es casi un lugar común hacer referencia a los planteamientos biopsicosociales en los discursos oficiales de la psiquiatría, [pero] a la hora de la verdad lo social sigue siendo la cenicienta de la psicopatología» (Achotegui, 2008: 16).

La Educación recomienda a los sanitarios:

- Entender la salud de forma integral (modelo holístico), es decir, concebir a la persona como un todo que interacciona, constantemente, con el medio ambiente que le rodea. Los galenos han de aceptar que esa interacción se realiza desde las dimensiones espiritual, social, biológica e intelectual de cada persona.

- Formarse en las características culturales que definen a cada colectivo de desplazados por nacionalidades.

- Realizar estudios epidemiológicos centrados en su heterogeneidad, en sus riesgos de salud específicos, en sus actitudes ante la enfermedad y ante cada tipo de enfermedad (Florenzano, et al., 2018). Actuando así se constatará que 
las dolencias desarrolladas por las poblaciones afectadas por el Síndrome de Ulises guardan relación, en múltiples ocasiones, con su cultura originaria y con su lugar de nacimiento.

- Según Vázquez, (Coordinador del Grupo de Atención al inmigrante de la Sociedad Española de Medicina de Familia y Comunitaria, semFYC) hay que aprovechar cualquier contacto con el sistema sanitario para realizar actividades de prevención y promoción de la salud (citado en Todo en Salud (2012), www.todoensalud/lacaraocultadelsindromedeulises).

- Con el objetivo de que el personal sanitario se convierta en un actor educativo eficaz, sería importante dotar a todos los sanitarios de una clave informática para acceder a la historia clínica de cada uno de estos pacientes. De esta forma todos los médicos del país podrían conocer todas las pruebas diagnósticas que se les han realizado así como los resultados obtenidos. Este dato facilitaría la actuación en red con idéntico criterio socioeducativo de todos los sanitarios.

- Contar con la colaboración de mediadores interculturales e intérpretes para poder entender el padecimiento real de estos enfermos.

- Realizar, sistemáticamente, videoconferencias entre médicos de familia especializados en Síndrome de Ulises para tratar historias clínicas de pacientes atendiendo, no solo a sus dolencias, sino también y sobre todo a sus nacionalidades.

- Mantener activa la red Atenea, ya existente, (www.laredatenea.com) para dar apoyo psicosocial a los desplazados en situación extrema por parte de psiquiatras españoles y extranjeros.

\section{Metodología de intervención socioeducativa: trato empático hacia el desplazado desolado}

El proceso socializador de todo individuo, para ser efectivo, ha de partir de su conocimiento intrínseco y de las necesidades ambientales que le rodean. Esta afirmación se torna fundamental en la socialización de las poblaciones desplazadas. Es un hecho que los desplazados vulnerables al Síndrome de Ulises viven en una cultura que perciben como hostil por el desconocimiento de las normas sociales, la relación entre causa y efecto de los hechos realizados y el nerviosismo que este desconocimiento les genera. El habitante autóctono conoce las relaciones entre causas y efectos sociales de cada acción desplegada. Este conocimiento innato lo aprovechará para seguir las normas de la hipótesis del contacto cultural Amir (1969) para normalizar la vida del desplazado.

La naturalidad y salubridad del contacto cultural, recalcaba Amir dependerá de las condiciones en las que se realice la interrelación poblador foráneo-autóctono. Una de las estrategias facilitadoras de este contacto cultural se basa en la escucha atenta, activa y empática de sentimientos e informaciones ofrecidas por el desplazado. Esa empatía se consigue, en este caso:

- Tratando a desplazados del mismo sexo que el ciudadano autóctono. Además del mismo sexo sería ideal compartir estatus cultural similar (el desplazado, al abandonar su país, suele perder su estatus social pero no así su estatus cultural). Establecer en el país de acogida relaciones sociales equiparables a las mantenidas en el país de origen rebaja su ansiedad. 
- Usando el mismo registro idiomático que utilice el desplazado (coloquial, vulgar, culto...). El desplazado percibe la cercanía o lejanía de su interlocutor dependiendo del registro idiomático que el nativo utilice.

- Escuchando al desplazado con apertura mental. Esta actitud abre una posible vía para establecer incipientes relaciones sociales.

- Manifestando cercanía afectiva a través del lenguaje no verbal, a través de los gestos y la sonrisa. Es, fundamentalmente, a través de los ojos, la boca y el tono de voz como la psicología considera que las personas adivinan y transmiten, claramente, los sentimientos propios y ajenos.

- Permitiendo, respetuosamente, la aparición del llanto tanto en hombres como en mujeres y justificando, al inmigrante, la naturalidad de su llanto.

- Interesándose, respetuosamente, por las vivencias contadas por el desplazado. Será preciso no invadir ni su intimidad ni forzándole a narrar aspectos que el desplazado no quiera contar.

- Imbuyéndose en la cultura del desplazado y desde esa visión cultural orientar la relación con él.

- No afeando conductas impropias (mentiras descubiertas). Es mejor que las rectificaciones las haga, el desplazado, por propia iniciativa.

El desplazado rebaja el nivel de ansiedad y comienza a relajarse (superando los estresores uno, tres y cuatro). La relajación abre las puertas a una incipiente comunicación. La primera ayuda que necesita un inmigrante pasa por dejarle hablar - señala-Castro (2010: 62). Al narrar sus experiencias se hace visible, al menos, para el interlocutor que le escucha. Es decir, abandona el anonimato, su invisibilidad para la sociedad del país de acogida y comienza a superar el primer estresor.

\section{Alfabetización emocional y toma de decisiones}

El desplazado obligado a vivir solo, sin una red social de apoyo y desconfiando de las intenciones de sus allegados suele presentar escasa o nula capacidad para conocer y, menos todavía, para comprender sus propios sentimientos o emociones. Siguiendo a Salovey, Mayer y Caruso (2002) y, posteriormente, a Goleman (2001) el desplazado no regularizado sufre un nuevo agravante del Síndrome de Ulises debido a su profundo analfabetismo emocional.

El analfabeto emocional es un ser que no ha podido, no ha sabido o no ha tenido la oportunidad de desarrollar dos tipos de inteligencias contenidas en la inteligencia personal: la inteligencia interpersonal y la inteligencia intrapersonal. El iletrado emocional percibe o procesa la información de una forma nada perspicaz y, paralelamente, procesa sus sentimientos de la misma forma.

El constructivismo, tradicionalmente, ha mantenido que la realidad no existe de manera independiente al observador. Es por ello por lo que el desplazado vive la realidad (ya no como es) si no como él la observa y la siente y esas erróneas percepciones le sumen en un profundo abatimiento. Para prevenir ese abatimiento es preciso alfabetizar 
emocionalmente al desplazado vulnerable. El primer paso consistirá en cubrir sus necesidades materiales. Después y solamente después de cubrir sus necesidades se le facilitará el desarrollo de su inteligencia inter e intrapersonal. Es preciso enseñar al desplazado a conocerse, a controlarse y a motivarse entendiendo los sentimientos de sus convecinos y acogiéndolos, también él, empáticamente.

El desplazado solo y abatido se alfabetizará emocionalmente, es decir, «será capaz de entender tanto las emociones y los sentimientos propios, como ajenos» (Segura, y Arcas, 2007: 30), en la medida que reconozca los sentimientos o las emociones que se producen en su persona y en los demás y conduzca esos sentimientos a través de técnicas básicas de control emocional (Segura y Arcas, 2007). La instrucción emocional mantenida en el tiempo y el control emocional desembocarán, antes o después, en un conjunto de capacidades, competencias y habilidades no cognitivas que influirán en una habilidad específica dirigida a afrontar las demandas y presiones ambientales de forma exitosa (Mayer, 2001), es decir, en el desarrollo de un nivel de inteligencia emocional.

Goleman (2001) argumenta que las personas emocionalmente desarrolladas, es decir, las personas que gobiernan adecuadamente sus sentimientos y que también saben interpretar y relacionarse con los sentimientos de los demás, suelen disfrutar de una situación ventajosa en todos los dominios de la vida. En la misma línea apostillan Vivas y Gallego (2008):

«Esas personas suelen sentirse más satisfechas, son más eficaces y capaces de dominar los hábitos mentales que determinan la productividad. Quienes por el contrario no controlan su vida emocional se debaten en constantes luchas internas que socavan su capacidad de trabajo y les impiden pensar con suficiente claridad» (p. 33).

El objetivo del desplazado que ha abandonado su país es disfrutar de seguridad. Este objetivo podrá irlo cumpliendo, entre otros factores, en la medida que domine sus sentimientos más nefastos o destructivos y con ello combata el segundo estresor que agobia su desarrollo personal (el sentimiento de fracaso). Esta idea de fracaso nace en el desplazado al no disfrutar del tipo de seguridad buscada. No obstante, en la medida que el desplazado pueda alfabetizarse emocionalmente se incrementarán, colateralmente, sus posibilidades de acceso al mercado laboral y este acceso permitirá comenzar a sentir seguridad.

Uno de los mecanismos que llevan a reducir la incidencia de este sentimiento de fracaso reside en el deseo y el ejercicio efectivo de una auténtica alfabetización emocional a través del desarrollo de habilidades de inteligencia emocional similares a las indicadas por Gallego, Alonso, Cruz y Lizama (1999:29). 
Tabla 1.

Habilidades de inteligencia emocional

\begin{tabular}{lll}
\hline Habilidades emocionales & Habilidades cognitivas & Habilidades conductuales \\
\hline $\begin{array}{l}\text { Reconocer, identificar y expre- } \\
\text { sar sentimientos }\end{array}$ & $\begin{array}{l}\text { Comprender los sentimien- } \\
\text { tos de los demás, asumir sus } \\
\text { puntos de vista y respetar las } \\
\text { diferencias }\end{array}$ & $\begin{array}{l}\text { Resistir las influencias nega- } \\
\text { tivas }\end{array}$ \\
\hline $\begin{array}{l}\text { Controlar sentimientos y eval- } \\
\text { uar su intensidad }\end{array}$ & $\begin{array}{l}\text { Saber leer e interpretar indica- } \\
\text { dores sociales }\end{array}$ & $\begin{array}{l}\text { Escuchar a los demás y re- } \\
\text { sponder, eficazmente a las } \\
\text { críticas }\end{array}$ \\
\hline $\begin{array}{l}\text { Aprender a controlar la an- } \\
\text { siedad, la tristeza, la ira y la } \\
\text { cólera }\end{array}$ & $\begin{array}{l}\text { Dividir en fases la toma de } \\
\text { decisiones y la resolución de } \\
\text { problemas }\end{array}$ & $\begin{array}{l}\text { Comunicarse con los otros a } \\
\text { través de canales no verbales } \\
\text { (gestos, tono de voz, expresión } \\
\text { facial...) }\end{array}$ \\
\hline $\begin{array}{l}\text { Controlar las emociones y } \\
\text { comprender lo que hay tras un } \\
\text { sentimiento }\end{array}$ & $\begin{array}{l}\text { Adiestrarse en la cooperación, } \\
\text { la resolución de conflictos y la } \\
\text { negociación de compromisos }\end{array}$ & $\begin{array}{l}\text { Participar en grupos positivos } \\
\text { de compañeros }\end{array}$ \\
\hline
\end{tabular}

Fuente: Elaboración propia a partir de Gallego, Alonso, Cruz y Lizama (1999).

El desarrollo de estas habilidades no es nada fácil de conseguir. Está influenciado por la cultura de origen del desplazado y por la forma de enfrentar el fracaso.

La Educación tiene que enseñar a estas posibles poblaciones dianas del Síndrome de Ulises a conocer qué emociones experimentan y por qué las experimenta, a percibir los vínculos entre sus sentimientos y lo que piensan, hacen y dicen, conocer sus puntos fuertes y débiles, ser reflexivos y mostrarse seguros de sí, controlar su impulsividad y las emociones perturbadoras que vayan sintiendo, pensar con claridad y no perder el control en situaciones de presión, ser empáticos, es decir, personas socialmente equilibradas que comprendan los sentimientos, las preocupaciones y las perspectivas de sus convecinos.

Todo esto puede intentar conseguirse a través de:

- Un ejercicio personal de contraste dirigido con el que se recuerden los verdaderos motivos que empujaron a cada una de las personas a salir de sus países. Ese ejercicio analítico servirá para rebajar el Síndrome de Ulises cuando se produzca. Ese contraste sociológico debe repetirse, periódicamente. Se realizará de manera espontánea o dirigida por un profesional sopesando inconvenientes y beneficios pasados, presentes y futuros. Esta introspección servirá, entre otras cosas para afrontar, activamente, la toma de decisiones diarias y personales. Una estrategia desarrollada por Quicios y Ruano con mujeres latinas para desplegar esta acción evaluadora consiste en la conexión permanente con la realidad socioeconómica del país de origen a través del ciberespacio. El uso cotidiano de Internet, chats, radio internacional y televisión por cable permite conocer, evaluar, sopesar y decidir, día a día, la orientación de las emocionales vividas enfrentando cotidianeidad próxima con realidad diaria del país de origen.

- Programa de ocio dirigido, esta estrategia ayuda a superar las frustraciones, ayuda a encontrar un espacio de esparcimiento fuera de la angustia vital y laboral, sirve para generar espacios amplios de relajación y disfrute a la vez 
que educa para enfrentar realidad perdida y realidad vivida. Esa confrontación de realidades puede realizarse de múltiples formas. Una de ellas es la realizada a través de las tics puesto que el proyecto de investigación I+D SEJ 2004-06803 I+D ha concluido que las herramientas virtuales trasladan a las realidades no tangibles de forma vivencial con auténtica credibilidad. Ese sentimiento de realidad ubicua, sincrónica, coloca al desplazado ante la realidad que decidió abandonar y la realidad que eligió. Le somete a la disyuntiva de elegir opción vital mientras le permite disfrutar de las ventajas afectivas de ambas situaciones y ambas sociedades. El ocio dirigido a través de tics permite superar los estresores uno y dos y decidir libremente qué opción vital elegir. A su vez permite ver la necesidad de redirigir el camino emprendido hacia el retorno o hacia la rudeza esperanzada de la realidad española.

En definitiva, el camino para superar el Síndrome de Ulises desde la actuación educativa, con unas u otras estrategias pasa por sopesar la opción vital elegida, valorar objetivamente la realidad socioafectiva personal que acompaña el diario devenir en el país de acogida, tomar una decisión, y ejecutarla de manera individual o auxiliado por las políticas sociales del país, si es que la situación personal del desplazado desolado no le permite actuar de manera autónoma. Mientras estas acciones se van llevando a cabo lo más indicado es gestionar el nivel de estrés acumulado y combatir la ansiedad o depresión de manera autónoma o asistida.

\section{7. $\quad$ Referencias}

Achotegui, J. (2000). Los duelos de la migración: una perspectiva psicopatológica y psicosocial. En E. Perdiguero y J. M. Comelles (comp.), Medicina y cultura, (88100). Barcelona: Editorial Bellaterra.

_(2002a). La depresión en los inmigrantes. Una perspectiva transcultural. Barcelona: Mayo.

_(2002b). Trastornos afectivos en los inmigrantes: la influencia de los factores culturales. Suplemento Temas candentes. Barcelona: Jano.

_ (2003a) (compilador). Dossier de la reunión internacional sobre el Síndrome de Ulises celebrada en Bruselas en la sede del Parlamento Europeo el 5 de noviembre del 2003.

_(2004a) (compilador). Dossier del Diálogo sobre el Síndrome de Ulises del Congreso Movimientos humanos y migración del Foro Mundial de las Culturas. Barcelona: 2-5 de septiembre del 2004.

_ (2004b). Emigrar en situación extrema. El Síndrome del inmigrante con estrés crónico y múltiple (Síndrome de Ulises). Revista Norte de salud mental de la Sociedad Española de Neuropsiquiatría, 5(21), 39-53.

_ (2008). Migración y crisis: El síndrome del inmigrante con estrés crónico y múltiple, síndrome de Ulises. ASMR Revista Internacional On-Line, 7(1), 1579-3516. 
_ (2009). Estrés límite y salud mental: el síndrome del inmigrante con estrés crónico y múltiple (Síndrome de Ulises) Gac Med Bilbao, 106, 122-33. Recuperado de: http://dev.elsevier.es/esrevista-gaceta-medica-bilbao-316avance-resumen-estres-limitesalud-mental-el-So304485809746657

_ (2017). El síndrome del inmigrante con estrés crónico y múltiple (síndrome de Ulises). Revista de Menorca, 96, 103-111.

Achotegui, J., Lahoz, S., Marxen, E. y Espeso, D. (10-15 de Septiembre 2005). Study of 30 cases of inmigrantes with The Immigrant Sindrome with Chronic and Multiple Stress (The Ulysses Syndrome). Comunication in the XVIII' ${ }^{\circ}$ World Congress of Psychiatry, Cairo, Egipto.

Achotegui, J., López, A., Morales, M., Espeso, D. y Achotegui, A. (2015). Estudio sociodemográfico de los inmigrantes con síndrome de Ulises. Estudio sobre 1.110 inmigrantes atendidos en el SAPPIR en Barcelona. Norte de salud mental, XIII, $52,70-78$.

Achotegui, J. y Jayme, M. (2016). El reto de trabajar la relación terapéutica extendida y la transferencia negativa con inmigrantes, minorías y excluidos sociales. Clínica Contemporánea, 7(2), 105-112.

Amir, Y. (1969). Contact hypothesis in ethnic relation. Psychological Bulletin, 71, 319-342.

Berry, J. W. (1980). Acculturation as varieties of adaptation. En A. Padilla (Ed.), Acculturation: Theory, models and some new findings. Boulder, CO: Westview.

_(1990). Psychology of acculturation: understanding individuals moving between cultures. En R. W. Brislin (ed.), Applied cross-cultural psychology. Newbury Park Calif.: Sage.

_(1997). Inmigration, acculturation, and adaptation. Applied Psychology, 46, 5-68.

Castro, Á. (2010). S. O. S....Soy inmigrante. El Síndrome de Ulises. Madrid: Pirámide.

Chávez,G. C. y Alava, L. (2019). Intervención del trabajador social frente a la inmigración, caso de estudio: familias venezolanas que residen en los bloques multifamiliares de la Ciudadela de los Tamarindos de la Ciudad de Portoviejo. Revista Caribeña de Ciencias Sociales. Recuperado de https://www.eumed.net/rev/caribe/2019/o3/ trabajador-social-inmigracion

Delgado, S. (2013). El síndrome de Ulises: El impacto psicológico de la emigración. Psicología en la Red. Recuperado de http://www.psicologiaenlared.com/elsindrome-de-ulises-el-impacto-psicologico-de-la-emigracion

Espar, M. (14 de junio de 2008). Síndrome de Ulises, la enfermedad de los invisibles. El País. Portada. La salud de los inmigrantes. Recuperado de https://elpais.com

Espeso, D. (2009). Estrés crónico y múltiple (Síndrome de Ulises) en población infantil, desde una perspectiva multicultural. Desenvolupa, 30. Recuperado de http://www. desenvolupa.net/Numeros-anteriors/Numero-30/Estres-cronico-y-multipleSindrome-de-Ulises-en-poblacion-infantil-desde-una-perspectiva-multiculturalDra.-Dori-Espeso-Montagud 
Florenzano, R., Bortolaso, M., Apablaza, M., Estay, P., Bustos, P., Lomeli, K. y Puig, G. (2018). Salud mental de migrantes consultantes en la unidad de enlace psiquiátrico de un hospital general: un análisis retro y prospectivo. Gaceta de Psiquiatría Universitaria, 14(2), 143-149.

Gallego-Díaz, S. (20 de septiembre de 2011). No es depresión, es Síndrome de Ulises. El País. Madrid.Sociedad. Recuperado de https://elpais.com/sociedad/2011/o9/20/ actualidad/1316469615_850215.html

Gallego, D., Alonso, C., Cruz, A. y Lizama, L. (1999). Implicaciones educativas de la inteligencia emocional. Madrid: UNED.

García Carrasco, J. (2006). ¿Cómo se forman los sentimientos? En A.A.V.V. Comunicaciones al $V$ Congreso Internacional de Filosofía de la Educación (1726). Madrid: Dykinson.

González de la Rivera, J. L. (Octubre de 2005). Migración, cultura, globalización y salud mental. II Jornadas de salud mental y medio ambiente. Lanzarote.

Goleman, D. (2001). Emotional Intelligence: Issues in Paradigm Building. En Cherniss, C. y Goleman, D. (Eds.), The emotionally intelligent workplace. San Francisco: Jossey-Bass.

_(2003). Emociones destructivas. Cómo entenderlas y superarlas. Barcelona: Kairos.

Huesca, A. (2009). El “derecho a la seguridad” en la base del desarrollo. Miscelanea, 67, 75-96.

_(2016). Seguridad Humana y pobreza. En S. Giménez, y D. Santos (Ed.) Integraciones y desintegraciones sociales. Pobreza, migraciones, refugio (335-350). Toledo: Asociación Castellano Manchega de Sociología.

Huesca, A., Giménez, S., y Quicios, M. P. (2018). Mejorando la seguridad de los refugiados: una propuesta de intervención del Trabajo Social clínico. Cuadernos De Trabajo Social, 31(1), 69-83. DOI: 10.5209/CUTS.56491

Lara, A., Jimenez, F., Tocornal, M.C., Cayupan, E., Gallardo, I., Mateluna, D. y Alcaino, M. (2017). Nudos críticos en la atención psicoterapéutica a migrantes y refugiados desde un centro universitario. Revista Sul Americana de Psicología, 5(2). 161-171.

Lara, A. (2019). Perspectiva de atención psicoterapéutica a personas en situación de refugio. Revista Interdisciplinar Mobilidad Humana 27(55), 97-112.

Mayer, J.D. (2001). Afield guide to emotional intelligence. En L. Feldman Barret y P. Salovery (Eds.), The wiadom of feelings: Psychological processes in emotional intelligence. New York: Guilford.

Moreno, J. (2006). La mediación en el ámbito de la inmigración y convivencia intercultural. Acciones e investigaciones sociales, 1, 113-192.

Navarro, M. (2015). La medicina emocional. Madrid: Vivelibro. 
Navarro-Lashayas, M.A. (2018). Población inmigrante sin hogar y salud mental. Cuaderno Psiquiatría Comunitaria, 15(1), 63-79.

Oberg, K. (1960). Cultural shock: Adjustment to New Cultural. Environments Practical Anthropology, 7, 177-182.

Quercetti, F. (29 de noviembre a 2 de diciembre de 2017). El derecho a la salud mental de las personas refugiadas y solicitantes de asilo. Psicopatologización del sufrimiento psíquico: el Síndrome de Ulises ¿una alternativa a las nosografías psiquiátricas clásicas? En N. Cervone (Presidente), Psicología, Culturas y Nuevas Perspectivas. IX Congreso Internacional de Investigación y Práctica Profesional en Psicología, Buenos Aires, Argentina.

Quicios, M. P. (Diciembre de 2008). Prevención del Riesgo Social o Síndrome de Ulises a través de la inteligencia emocional. II Congreso Internacional de Inteligencia Emocional. Las Palmas de Gran Canaria.

Quicios, M. P. y Quicios, M. (2010). La otra cara de la inmigración: educación para el retorno. Revista Iberoamericana de Educación. EOI, 51(3), 1-13.

Real Decreto Ley 4/2008, de 19 de septiembre, sobre abono acumulado y de forma anticipada de la prestación contributiva por desempleo a trabajadores extranjeros no comunitarios que retornen voluntariamente a sus países de origen. Boletín Oficial del Estado. Madrid, 20 de septiembre de 2008, núm. 228, pp. 44727 a 44729.

Real Decreto 1800/2008, de 3 de noviembre, por el que se desarrolla el Real Decreto-ley 4/2008, de 19 de septiembre, sobre abono acumulado y de forma anticipada de la prestación contributiva por desempleo a trabajadores extranjeros no comunitarios que retornen voluntariamente a sus países de origen. Boletín Oficial del Estado. Madrid, 11 de noviembre de 2008, núm.272, pp. 44727 a 44729.

Reig-Botella, A., Clemente, M. y Sangiao, I. (2018). Migración y Síndrome de Ulises: ser nadie en tierra de nadie. Barataria, Revista Castellano-Manchega de Ciencias Sociales, 24, 27-43.

Rodríguez, O. (1 de marzo de 2011). Síndrome de Ulises, el sufrimiento de los inmigrantes. En la Web para TODOS los inmigrantes, www.tomai.es

Salaberria, K. y Del Valle, A. (2017). Estrés migratorio y salud mental. Behavioral Psychology/Psicología Conductual, 25(2), 419-432.

Salovey, P., Mayer, J.D., y Caruso, D. (2002). The positive psychology of emotional intelligence. In C.R. Snyder y S.J. Lopez (Eds.), The handbook of positive psychology (159-171). New York: Oxford University Press.

Segura, M. y Arcas, M. (2007). Educar las emociones y los sentimientos. Introducción práctica al complejo mundo de los sentimientos. Madrid: Narcea.

Sevillano, M. L. (Dir.) (2010). Diagnóstico y desarrollo de competencias en el uso de herramientas de comunicación virtuales para la sociedad del conocimiento a lo largo de la vida. Madrid: Pearson. 
Todo en Salud (2012). http://todo-en-salud.com/2010/12/la-cara-oculta-del-sindromede-ulises

Vallejo-Sánchez, B. y Pérez-García, A. M. (2016). Estrés vital: variables psicológicas y sociodemográficas predictoras del malestar emocional. Acción Psicológica, 13(1), 159-178. DOI: 10.5944/ap.13.1.16150

Vallés, A. y Vallés, C. (2003). Psicología de la inteligencia emocional. Valencia: Promolibro.

Vauclaire, C.M. y Bratanova, B. (2016). Income inequality and fear of crime across the European region. European Journal of Criminology, (2) 221-241. DOI: $0.1177 / 1477370816648993$

Vázquez, J. (Octubre de 2005). Estudio de la salud mental de una población en un área de atención primaria en Almería. VIII Congreso de la Asociación Andaluza de Neuropsiquiatría, Almería.

Vivas, M. y Gallego, D. (2008). La inteligencia emocional ¿̇Por qué y cómo desarrollarla? Mérida: Consejo de Publicaciones. Universidad de los Andes.

Vivas, M., Gallego, D. y González, B. (2006). Educar las emociones. Madrid: Dykinson. 